\title{
Molecular Cloning and Characterization of the HOS1 Gene from 'Muscat Hamburg' Grapevine
}

\author{
Jitao Li ${ }^{1}$ \\ Key Laboratory of Plant Germplasm Enhancement and Specialty Agriculture, Wuhan Botanical \\ Garden, Chinese Academy of Sciences, Wuhan 430074, China; and the University of Chinese \\ Academy of Sciences, Beijing 100049, China \\ Nian Wang ${ }^{1}$ \\ Key Laboratory of Plant Germplasm Enhancement and Specialty Agriculture, Wuhan Botanical \\ Garden, Chinese Academy of Sciences, Wuhan 430074, China \\ Lina Wang \\ Key Laboratory of Plant Germplasm Enhancement and Specialty Agriculture, Wuhan Botanical \\ Garden, Chinese Academy of Sciences, Wuhan 430074, China; and the University of Chinese \\ Academy of Sciences, Beijing 100049, China \\ Haiping Xin ${ }^{2}$ \\ Key Laboratory of Plant Germplasm Enhancement and Specialty Agriculture, Wuhan Botanical \\ Garden, Chinese Academy of Sciences, Wuhan 430074, China \\ Shaohua $\mathbf{L i}^{\mathbf{2}}$ \\ Key Laboratory of Plant Germplasm Enhancement and Specialty Agriculture, Wuhan Botanical \\ Garden, Chinese Academy of Sciences, Wuhan 430074, China; and Beijing Key Laboratory of Grape \\ Sciences and Enology, CAS Key Laboratory of Plant Resources, Institute of Botany, Chinese Academy \\ of Sciences, Beijing, 100093, China
}

\begin{abstract}
AdDitional INDEX words. abiotic stress, Vitis vinifera, VvHOS1
Abstract. Cold stress is an important factor that limits grape (Vitis sp.) production around the world. The high expression of osmotically responsive genes $1(\mathrm{HOS} 1)$ protein acts as a repressor of cold-responsive genes in plants. To increase understanding of mechanism regulating cold tolerance in grape, we isolated and characterized a novel $\mathrm{HOS} 1$ gene, designated $V v H O S 1$ from 'Muscat Hamburg' grapevine (Vitis vinifera). Real-time polymerase chain reaction (PCR) analysis revealed that the expression of $V v H O S 1$ could be induced by the application of exogenous abscisic acid and various abiotic environmental conditions such as low temperature, drought, and salinity. Moreover, VvHOS1 expression could also be induced by cold plus drought conditions $\left(4{ }^{\circ} \mathrm{C}, 10 \%\right.$ polyethylene glycol 6000$)$. In addition, overexpression of $\mathrm{VvHOS} 1$ in arabidopsis (Arabidopsis thaliana) decreased the plants' tolerance to cold, drought, and salt as well as negatively regulated the expression level of two stress-responsive genes, $A t R D 29 A$ and $A t C O R 47$. The results obtained in this study should help us to elucidate the function of $V v H O S 1$ and understand the cold-responsive pathway in grapevine.
\end{abstract}

Cold stress, including chilling $\left(0\right.$ to $\left.15^{\circ} \mathrm{C}\right)$ and freezing (less than $0^{\circ} \mathrm{C}$ ), is one of the limiting environmental factors affecting plant growth (Levitt, 1980). To adapt to a cold environment, many plants have evolved a cold acclimation process when exposed to low non-freezing temperatures mediated by complex and elaborate signaling networks (Guy, 1990; Tang et al., 2006; Thomashow, 1999). This process is associated with the accumulation of compatible osmolytes and the stability of biomembranes (Orvar et al., 2000; Suzuki et al., 2000; Suzuki and Mittler, 2005). For the past several years, considerable attention has been devoted to the transcriptional activation of

Received for publication 15 May 2013. Accepted for publication 15 Oct. 2013. This work was funded by the National Natural Science Foundation of China (NSFC accession no. 31000902 and 31130047) and a CAS special grant for postgraduate research, innovation, and practice.

${ }^{1} \mathrm{Jitao} \mathrm{Li}$ and Nian Wang have the same contribution.

${ }^{2}$ Corresponding authors. E-mail: xinhaiping215@hotmail.com; shhli@ wbgcas.cn. the positive cold-responsive genes such as C-repeat binding factor $(C B F)$ and its downstream genes: responsive to dessication 29A (RD29A), cold-regulated 15A (COR15A), cold-regulated 47 (COR47), etc. A large number of genes that respond to cold acclimation have been identified in a number of plants (Fursova et al., 2009; Ganeshan et al., 2008; Knight et al., 2009; Lee and Thomashow, 2012; Medina et al., 2011; Provart et al., 2003; Thomashow, 2010; Xiao et al., 2006). Water-deficit stress such as drought and high salinity results in a marked reduction in crop productivity on as much as half of the irrigated land in the world. Signal transduction pathways triggered by various stresses, including drought and high salt content, share a number of signaling components that transduce the signal into downstream processes, which subsequently endow resistance to such stresses. In contrast, continuous activation of the plant's cold-responsive genes is metabolically expensive and may result in permanent damage to the cellular components of the plant itself; thus, the expression of the cold-responsive genes must be controlled by negative regulation to maintain the balance of gene expression in 
the plant. Transcriptional repression and negative regulation are also important components of the cold-response regulatory cascades (Thiel et al., 2004). Therefore, the investigation of negative regulatory factors is extremely important in elucidating these pathways.

Components of the CBF-dependent signaling pathway are negatively regulated by upstream transcription factors. A negative regulator of this pathway, HOS1, was identified in arabidopsis. Plants with a mutated form of this gene, Hos 1-1, exhibit enhanced expression of the cold-responsive genes (Ishitani et al., 1998). HOS1 encodes an E3 ubiquitin ligase containing a RING-finger motif, and this gene product regulates both cold acclimation and vernalization in arabidopsis (Lazaro et al., 2012). HOS1 protein is localized in the cytoplasm at normal growth temperatures but translocates to the nucleus in response to cold treatments, thereby causing the cold-regulated nucleocytoplasmic partitioning activity that negatively regulates cold signal transduction (Lee et al., 2001). During cold acclimation, HOS1 mediates the ubiquitination and degradation of ICE1 to reduce the $\mathrm{CBF}$-induced activation of many cold-responsive genes and thus to decrease the plant's cold tolerance (Dong et al., 2006). In fact, ICE1 has been identified as a target of the HOS1 ubiquitin ligase. $I C E 1$, the upstream transcriptional activator of $C B F$, can control the expression of $C B F$ and its downstream cold-responsive genes in arabidopsis (Chinnusamy et al., 2003; Zarka et al., 2003). The AtICE1 gene encodes a MYC basic helix-loop-helix (bHLH) transcription activator, which binds to the MYC cis-elements (CANNTG) in the promoter of the $C B F 3$ gene to induce its expression (Miura et al., 2007). However, ICE1 has little effect on the cold induction of the $C B F 1$ and $C B F 2$ genes. Furthermore, HOS1 also negatively regulates vernalization and flowering time by decreasing FLOWERING LOCUS C (FLC) expression (Ishitani et al., 1998; Lazaro et al., 2012), which is a central repressor of flowering induction by vernalization (Michaels and Amasino, 1999; Sheldon et al., 2000). Overexpression of the U-box-containing E3 ubiquitin ligase gene AtCHIP in arabidopsis rendered plants more sensitive to both low- and high-temperature treatments (Yan et al., 2003). The arabidopsis DREB2A-interacting proteins, DRIP1 and DRIP2, function as RING E3 ubiquitin ligases and negatively regulate drought stress-responsive gene expression by marking DREB2A to be degraded by the $26 \mathrm{~S}$ proteasome (Qin et al., 2008). Another RING domain E3 ubiquitin ligase, AtRGLG2, negatively regulates the drought stress response by mediating the transcriptional activity of AtERF53 in arabidopsis (Cheng et al., 2012). Overexpression of the arabidopsis RING-H2 gene XERICO results in hypersensitivity to salt and osmotic stresses and exogenous abscisic acid (ABA) during germination and early seedling growth (Ko et al., 2006). To gain insight into the biotic and abiotic stress resistance mechanisms in grape, in this study, we isolated and characterized a novel HOS1 gene and investigated its response to various abiotic stresses.

Grapevine is the most widely planted fruit crop worldwide and is cultivated in all continents except Antarctica (Mullins et al., 1992). However, cold stress limits the geographical distribution and productivity of grape and results in considerable economic losses. As one of the most important cultivars, Muscat Hamburg is largely cultivated in the world as a result of its interesting fruit quality. Although cold-hardiness is found in 'Muscat Hamburg', which can withstand midwinter temperatures to $-22{ }^{\circ} \mathrm{C}$ (Fennell, 2004), these plants need to be buried in soil to protect the vine in winter in northern China, where the minimal temperature in winter is much higher than the previous critical temperature and this burial is an obligatory practice for all the cultivars of $V$. vinifera. This phenomenon should be explained by low temperature plus dry climate in winter. Therefore, the molecular mechanisms of cold tolerance are important for the grape industry. To increase our understanding of the molecular mechanism of this plant's response to cold stress, it is necessary to confirm the molecular nature of the cold-responsive genes and to elucidate the regulatory network. The identification of genes that regulate cold acclimation in grape cultivars could be useful for molecular breeding. In this study, we isolated the VvHOS1 gene from 'Muscat Hamburg' and characterized its expression patterns under various abiotic stress conditions and after the application of exogenous ABA. It was also shown that ectopic overexpression of $V v H O S 1$ in transgenic arabidopsis plants resulted in decreased tolerance to cold, drought, and salt.

\section{Materials and Methods}

Plant materials. Tissue culture seedlings of 'Muscat Hamburg', the clonal plants germinated and grown in vitro, were cultured on half-strength B5 basal medium (Gamborg et al., 1968 ) containing $30 \mathrm{~g} \cdot \mathrm{L}^{-1}$ sucrose, $1.25 \mathrm{~g} \cdot \mathrm{L}^{-1}$ potassium nitrate $\left(\mathrm{KNO}_{3}\right), 12.5 \mathrm{mg} \cdot \mathrm{L}^{-1}$ inositol, and $0.2 \mathrm{mg} \cdot \mathrm{L}^{-1}$ indole-3-acetic acid in a growth chamber for 1 month at a constant temperature of $26^{\circ} \mathrm{C}$ under a cycle of $16 \mathrm{~h}$ of light with an intensity of $100 \mu \mathrm{mol} \cdot \mathrm{m}^{-2} \cdot \mathrm{s}^{-1}$ and $8 \mathrm{~h}$ of darkness.

RNA EXTRACTION AND FIRST-STRAND CDNA SYNTHESIS. Total RNA was extracted from the shoot apex with the first two young leaves of tissue culture seedlings of 'Muscat Hamburg' using a Column Plant RNAout 2.0 Kit (90404-50; Tiandz, Beijing, China). All protocols followed the manufacturer's instructions. The quality of the extracted RNA was determined by agarose gel electrophoresis, and the concentration was quantified using a spectrophotometer (Nanodrop ND-1000; Thermo Fisher Scientific, Waltham, MA). First-strand cDNA was synthesized from $1 \mu \mathrm{g}$ high-quality total RNA treated with DNaseI (RQ1; Promega, Fitchburg, WI) in a $20-\mu \mathrm{L}$ reaction volume using random hexamer primers and SuperScript III Reverse Transcriptase (Invitrogen, Carlsbad, CA) according to the manufacturer's protocols.

ACQUiSITION AND SEQUENCING OF THE FULL-LENGTH VVHOSI CDNA. AtHOS1 (At2g39810) was used to find the homologous fragment of the $V$. vinifera HOS1 gene in the Vitis genome, which is available in the Grape Genome Browser database (Genoscope, 2009). A pair of gene-specific primers P1 (Table 1) were designed based on this sequence to amplify this fragment. The fragment was amplified using the following program: one cycle of $30 \mathrm{~s}$ at $94{ }^{\circ} \mathrm{C}, 35$ cycles of $5 \mathrm{~s}$ at $94^{\circ} \mathrm{C}, 15 \mathrm{~s}$ at $54{ }^{\circ} \mathrm{C}, 20 \mathrm{~s}$ at $72{ }^{\circ} \mathrm{C}$, and one cycle of $2 \mathrm{~min}$ at $72{ }^{\circ} \mathrm{C}$. The resulting PCR product was separated on a $1.2 \%$ agarose gel and extracted using a TIANgel Midi Purification Kit (Tiangen, Beijing, China). Purified fragments were then cloned into the pGEM T-easy vector (Promega) and transformed into Escherichia coli DH5 $\alpha$ cells. Positive colonies were confirmed through PCR analysis and sequencing of the inserted DNA (Sunny, Shanghai, China).

For the 3 '-RACE protocol, first-strand cDNA was reversetranscribed using a SMARTer ${ }^{\mathrm{TM}}$ RACE cDNA Amplification Kit (Clontech, Mountain View, CA) using $3^{\prime}$-CDS as a primer according to the manufacturer's instructions with some modifications. To obtain the $3^{\prime}$ end of the $V v H O S 1$ gene, $3^{\prime}$-RACE was 
Table 1. Primer sequences used in this study for polymerase chain reaction analysis in 'Muscat Hamburg' grapevine and arabidopsis.

\begin{tabular}{|c|c|c|c|}
\hline No. & Name & Oligonucleotides $\left(5^{\prime} \rightarrow 3^{\prime}\right)$ & Note \\
\hline P1 & HOS1f & gaactcatcctaagatcgcacaa & For acquisition of $V v H O S 1$ fragment from 'Muscat Hamburg' \\
\hline $\mathrm{P} 2$ & HOS1-3f1 & ttacggtggtctgggcatgatggg & Nested primers for $3^{\prime}$ RACE of $V v H O S 1$ \\
\hline P4 & HOS1-5r1 & caccaagccctccggatacaaaga & \multirow[t]{2}{*}{ Nested primers for $5^{\prime} \mathrm{RACE}$ of $V v H O S 1$} \\
\hline P5 & HOS $1-5 \mathrm{r} 2$ & gtcaccagtgtctccatccaatcc & \\
\hline $\mathrm{P}^{\mathrm{z}}$ & HOS1fs & aaacgagctcaacactcattcacacatttatcatt & For full-length cloning of $V v H O S 1$ from 'Muscat Hamburg' \\
\hline \multirow[t]{2}{*}{ P7 } & HOS1s & caaggaaaagcagttacggg & \multirow[t]{2}{*}{ For qRT-PCR analysis of $V v H O S 1$ in 'Muscat Hamburg' and transgenic arabidopsis } \\
\hline & HOS1a & ctctatcattctatccaccaagcc & \\
\hline \multirow[t]{2}{*}{ P8 } & 18SrRNAs & cgaaagcatttgccaaggat & \multirow[t]{4}{*}{ For qRT-PCR analysis of the reference genes in 'Muscat Hamburg' } \\
\hline & 18SrRNAa & ccctggtcggcatcgtttat & \\
\hline \multirow[t]{2}{*}{ P9 } & EF1 $1 \alpha s$ & gaactgggtgcttgataggc & \\
\hline & EF1 $\alpha \mathrm{a}$ & aaccaaaatatccggagtaaaaga & \\
\hline \multirow[t]{2}{*}{ P12 } & AtACTINs & cttgcaccaagcagcatga & \multirow{2}{*}{ For qRT-PCR analysis of transgenic arabidopsis } \\
\hline & AtACTINa & ccgatccagacactgtacttcctt & \\
\hline
\end{tabular}

${ }^{\mathrm{z}}$ The underlined bases in primers are the restriction sites.

qRT-PCR = quantitative reverse transcription-polymerase chain reaction.

performed according to the instructions of the SMARTer ${ }^{\mathrm{TM}}$ RACE Kit. Briefly, two nested gene-specific primers, P2 and P3 (Table 1), were designed according to the candidate $\mathrm{VvHOS1}$ gene fragment amplified previously. The UPM primer mix provided by the RACE kit, including $0.4 \mu \mathrm{M}$ UPML and $2 \mu \mathrm{M}$ UPMS, was used as an antisense primer during the first round of PCR. The $10 \mu \mathrm{M}$ NUP primer provided by the RACE kit was used as an antisense primer for the second round of PCR. The first-round PCR reaction was performed in a total volume of $50 \mu \mathrm{L}$, including $35.3 \mu \mathrm{L}$ double-distilled $\mathrm{H}_{2} \mathrm{O}, 5 \mu \mathrm{L} 10 \times$ Ex Taq buffer, $1 \mu \mathrm{L} 10 \mathrm{~mm}$ dNTP mix, $2.5 \mu \mathrm{L}$ diluted 3'RACE-ready cDNA, $5 \mu \mathrm{L}$ UPM primer mix, $1 \mu \mathrm{L} 10 \mu \mathrm{M}$ P2 primer, and $0.2 \mu \mathrm{L} 5 \mathrm{U} \cdot \mu \mathrm{L}^{-1} \mathrm{Ex}$ Taq DNA polymerase. The touch-down PCR program was one cycle of $30 \mathrm{~s}$ at $94{ }^{\circ} \mathrm{C}$, five cycles of $10 \mathrm{~s}$ at $94^{\circ} \mathrm{C}, 30 \mathrm{~s}$ at $69^{\circ} \mathrm{C}, 1.5 \mathrm{~min}$ at $72^{\circ} \mathrm{C}$, five cycles of $10 \mathrm{~s}$ at $94^{\circ} \mathrm{C}$, $30 \mathrm{~s}$ at $67^{\circ} \mathrm{C}, 1.5 \mathrm{~min}$ at $72^{\circ} \mathrm{C}, 20$ cycles of $30 \mathrm{~s}$ at $94^{\circ} \mathrm{C}, 10 \mathrm{~s}$ at $65{ }^{\circ} \mathrm{C}, 1.5 \mathrm{~min}$ at $72{ }^{\circ} \mathrm{C}$, and one cycle of $3 \mathrm{~min}$ at $72{ }^{\circ} \mathrm{C}$. Subsequently, the second-round PCR was carried out using a 50 -fold dilution of the first-round PCR product as a template and the NUP and P3 oligos (Table 1) as primers. The touch-down PCR program was one cycle of $30 \mathrm{~s}$ at $94^{\circ} \mathrm{C}$, five cycles of $10 \mathrm{~s}$ at $94{ }^{\circ} \mathrm{C}, 30$ s at $70^{\circ} \mathrm{C}, 1.5$ min at $72{ }^{\circ} \mathrm{C}$, five cycles of $10 \mathrm{~s}$ at $94{ }^{\circ} \mathrm{C}$, $30 \mathrm{~s}$ at $67^{\circ} \mathrm{C}, 1.5 \mathrm{~min}$ at $72{ }^{\circ} \mathrm{C}, 20$ cycles of $30 \mathrm{~s}$ at $94{ }^{\circ} \mathrm{C}, 10 \mathrm{~s}$ at $64{ }^{\circ} \mathrm{C}, 1.5 \mathrm{~min}$ at $72{ }^{\circ} \mathrm{C}$, and one cycle of $3 \mathrm{~min}$ at $72{ }^{\circ} \mathrm{C}$. The PCR product was separated on a $1.2 \%$ agarose gel. The purified fragments were cloned into the pGEM T-easy vector and then transformed into E. coli (DH5a) cells. White colonies were picked and the inserted DNA sequenced.

First-strand cDNA for the 5'-RACE reaction was synthesized using PowerScript Reverse Transcriptase (Clontech) using the $5^{\prime}$ linker (SMARTer IIA oligonucleotide) and the $5^{\prime}$ CDS primer provided by the RACE kit. Nested PCR was carried out using a Advantage 2 PCR Kit (Clontech). Two antisense gene specific primers, P4 and P5 (Table 1), were designed for the $5^{\prime}$-RACE reaction. Touch-down PCR was also implemented in both rounds of the PCR program. The purified product of the nested PCR reaction was sequenced. The detailed procedure was similar to that described for the $3^{\prime}$-RACE reaction.

The primer pair P6, including the sense primer HOS1fs located in the $5^{\prime}$ UTR ( $\mathrm{SacI}$ site is underlined) and the antisense primer HOS1rs located in the $3^{\prime} \mathrm{UTR}$ region (Sall site is underlined), was designed for reverse transcription PCR (RTPCR) (Table 1). The first-strand cDNA used for amplifying the candidate $V v H O S 1$ gene fragment was also used as a template to isolate the full-length cDNA of VvHOS1 from 'Muscat Hamburg'. The PCR reaction was carried out in a total volume of $50 \mu \mathrm{L}$, including $33 \mu \mathrm{L}$ double-distilled $\mathrm{H}_{2} \mathrm{O}, 10 \mu \mathrm{L} 5 \times \mathrm{HF}$ buffer, $1 \mu \mathrm{L} 10 \mathrm{~mm}$ dNTPs, $1 \mu \mathrm{L} 10 \mathrm{~mm}$ sense and antisense primers, $1.5 \mu \mathrm{L}$ DMSO, $2 \mu \mathrm{L}$ cDNA template, and $0.5 \mu \mathrm{L}$ $2 \mathrm{U} \cdot \mu \mathrm{L}^{-1}$ Phusion DNA Polymerase (NEB, Ipswich, MA). The PCR was amplified using the following program: one cycle of $30 \mathrm{~s}$ at $98^{\circ} \mathrm{C}, 35$ cycles of $5 \mathrm{~s}$ at $94{ }^{\circ} \mathrm{C}, 15 \mathrm{~s}$ at $56^{\circ} \mathrm{C}, 1.5 \mathrm{~min}$ at $72{ }^{\circ} \mathrm{C}$, and one cycle of 6 min at $72{ }^{\circ} \mathrm{C}$. The resulting PCR product was separated on $1.2 \%$ agarose gels. The purified positive fragments were cloned into the pGEM T-easy vector and then transferred into E. coli (DH5a). White colonies were picked out and the inserted DNA was sequenced.

Bioinformatics analysis. The verified full-length cDNA sequence was translated into amino acid sequences using the Expert Protein Analysis System server [ExPASy (Swiss Institute of Bioinformatics, 1993)]. The theoretical isoelectric point (pI) and molecular weight were also predicted by the ExPASy ProtParam tool. The bHLH-ZIP domain was predicted using the web-based protein database Simple Modular Architecture Research Tool [SMART (Schultz et al., 1998)]. Related protein sequences were retrieved from the GenBank database using the BLASTP algorithm at the National Center for Biotechnology Information (NCBI). VvHOS1 protein sequence was used as a query sequence and several homologous HOS1 proteins from different plants were selected based on their score. A multiple protein sequence alignment was performed using the ClustalW program (Thompson et al., 1994) using the default 
parameters. For phylogenetic and sequence alignment analysis, the HOS1-like protein sequences from other plant species were obtained from the GenBank database, including arabidopsis (NP_181511), Arabidopsis lyrata (XP_002879823), Populus trichocarpa (XP_002304293), Ricinus communis (EEF30910), Poncirus trifoliata (ACY92092), Oryza sativa (AFK10207), and Physcomitrella patens (XP_001780235). The phylogenetic tree generated using the HOS1 protein sequences was constructed using the neighbor-joining (NJ) method of the MEGA software [Version 4.0 (Tamura et al., 2007)].

Real-time PCR analysis. For the expression analysis, 1-month-old tissue culture seedlings, fully adapted to growing in a growth chamber at $26{ }^{\circ} \mathrm{C}$ under a 24-h light intensity of $100 \mu \mathrm{mol} \cdot \mathrm{m}^{-2} \cdot \mathrm{s}^{-1}$, were transferred to another chamber set at $4{ }^{\circ} \mathrm{C}$ with a constant light intensity of $100 \mu \mathrm{mol} \cdot \mathrm{m}^{-2} \cdot \mathrm{s}^{-1}$ for the cold treatment. Drought, salinity, and ABA treatments were initiated when the tissue culture plants were transferred from the solid half-strength B5 medium to a liquid half-strength B5 medium supplemented with $10 \%$ polyethylene glycol (PEG) $6000,100 \mu \mathrm{M} \mathrm{NaCl}$, or $100 \mu \mathrm{M}$ ABA, respectively. The plantlets were also placed in liquid half-strength $\mathrm{B} 5$ medium containing $10 \%$ PEG 6000 under $4{ }^{\circ} \mathrm{C}$ to mimic a cold plus drought environment. Samples of the shoot apex with the first two young leaves were collected at $0,0.5,1,2,4,8,24$, and $48 \mathrm{~h}$. Samples were immediately frozen in liquid $\mathrm{N}_{2}$ and stored at $-80{ }^{\circ} \mathrm{C}$ until analysis. For each tested tissue, three biological replicates were collected by harvesting samples from three different plants.

Total RNA was extracted using a Column Plant RNAout 2.0 Kit and digested with RNase-free DNaseI (RQ1) to eliminate the remaining traces of genomic DNA. First-strand cDNA was synthesized from $1 \mu \mathrm{g}$ total RNA in a $20-\mu \mathrm{L}$ reaction volume using a M-MLV Reverse Transcriptase Kit (Invitrogen). $V v H O S 1$ gene fragments were amplified using the gene-specific primer pair P7 (Table 1). The $18 S$ rRNA and EFl $\alpha$ genes (AF207053 and BQ799343, respectively) were used as reference genes in this analysis by qbaseplus software (Hellemans et al., 2007) and were amplified using primer pairs P8 and P9 (Table 1), respectively. The reaction mixture contained $5 \mu \mathrm{L}$ of $2 \times$ SYBR Green Master Mix (Roche, Basel, Switzerland), $2.6 \mu \mathrm{L}$ double-distilled $\mathrm{H}_{2} \mathrm{O}, 0.2 \mu \mathrm{L}$ each primer $(10 \mu \mathrm{M})$, and $2 \mu \mathrm{L}$ diluted cDNA template. The PCR was performed in a StepOne Plus real-time PCR instrument (Applied Biosystems, Foster City, CA) using the following conditions: one cycle of $5 \mathrm{~min}$ at $95^{\circ} \mathrm{C}, 40$ cycles of $95^{\circ} \mathrm{C}$ for $15 \mathrm{~s}, 60^{\circ} \mathrm{C}$ for $1 \mathrm{~min}$, and one cycle of $95^{\circ} \mathrm{C}$ for $10 \mathrm{~min}$. The fluorescent products were read at the last step of each cycle. To confirm the specificity of the primers, a melting curve was constructed after the final PCR cycle. Furthermore, the real-time PCR products were analyzed on a $1.2 \%$ agarose gel to confirm the presence of a single amplicon and were later sequenced. The three biological and technical replicates were performed. Reaction mixtures without cDNA templates were used as negative controls to evaluate the specificity of each real-time PCR. The $\mathrm{Ct}$ values and the realtime PCR efficiencies were obtained using LinRegPCR (Ruijter et al., 2009) and the normalized relative quantities and SEs for each sample were obtained by qbaseplus (Hellemans et al., 2007). The relative fold difference for $V v H O S 1$ expression in various abiotic condition was calculated on the basis of the normalized relative quantities obtained previously with the normalized relative quantity of the untreated samples as one. The $\Delta \mathrm{Ct}$ value for each sample was obtained by subtracting mean $\mathrm{Ct}$ ( VvHOS1) from mean $\mathrm{Ct}$ (reference), where mean $\mathrm{Ct}$ for $V v H O S 1$ is the average cycle number at which a reaction reaches a specified fluorescence level from all biological and technique replications. The relative expression data were analyzed using the Eq. $2^{-\Delta \Delta \mathrm{Ct}}$.

The expression levels of two endogenous stress response genes $A t R D 29 A$ and $A t C O R 47$ in two $\mathrm{T}_{4}$ transgenic lines and wild-type arabidopsis were examined using real-time PCR. For this purpose, the third and fourth rosette leaves were harvested from 4-week-old wild-type and $\mathrm{T}_{4}$ transgenic arabidopsis lines (OE1 and OE2) between 1100 and $1200 \mathrm{HR}$ (i.e., 3 to $4 \mathrm{~h}$ after starting the light period), frozen in liquid N2, and stored at $-80{ }^{\circ} \mathrm{C}$ pending RNA extraction. cDNA preparation and reaction conditions were as described previously. Real-time PCR was also conducted using a StepOnePlus qPCR machine and the SYBR GreenI Master Mix (Roche) with gene-specific primers for AtRD29A (P10, Table 1), AtCOR47 (P11, Table 1) and $V a C B F 4$ (cbf4s and cbf4a). The relative expression level of each gene was calculated on the basis of the arabidopsis reference gene AtACTIN with the primer pair P12 (Table 1). The expression level of each gene in the wild-type arabidopsis was set to 1 . For $V v H O S 1$, the amplification on $10 \mathrm{pg}$ plasmid DNA (pCAMBIA 1301s-35S-VvHOS1) was set to 1 .

VECTOR CONSTRUCTION AND ARABIDOPSIS TRANSFORMATION. The coding region of $V v H O S 1$ was amplified using the primer pair P6 (Table 1). The digested PCR product was ligated into the pCAMBIA 1301s vector, which is a modified form of the pCAMBIA1301 vector (donated by Y. Zhou), between the corresponding restriction sites under the control of the CaMV $35 \mathrm{~S}$ promoter. The recombinant plasmid was sequenced to confirm the correct insertion and electroporated into Agrobacterium tumefaciens strain GV3101 cells. The genetic transformation of arabidopsis was performed using the floral dip method (Clough and Bent, 1998). Transgenic arabidopsis plants were selected on solid half-strength Murashige-Skoog medium [MS (Murashige and Skoog, 1962)] containing $50 \mu \mathrm{g} \cdot \mathrm{L}^{-1}$ hygromycin $\mathrm{B} . \mathrm{T}_{1}$ transgenic plants were confirmed by RT-PCR analysis using the primer pair P6 (Table 1) for $V v H O S 1 . \mathrm{T}_{4}$ plants were used for the stress tolerance experiments.

Analysis of COLD, Drought, and SAlt tolerance in TRANSGENIC ARABIDOPSIS PLANTS. Abiotic stress tests were carried out as previously described (Dong et al., 2006) with some modifications. Arabidopsis seedlings (wild-type and $\mathrm{T}_{4}$ transgenic lines OE1 and OE2) were grown for $12 \mathrm{~d}$ on halfstrength MS medium in a growth chamber under a 16-h light $\left(22^{\circ} \mathrm{C}\right)$ and 8 -h dark cycle $\left(20^{\circ} \mathrm{C}\right)$. The seedlings were later transferred to half-strength MS medium containing different concentrations of PEG $6000(0 \%, 4 \%$, and $8 \%)$ or $\mathrm{NaCl}(0,20$, and $40 \mathrm{~mm}$ ) to assess drought and salt tolerance. The seedlings were subjected to stress for $5 \mathrm{~d}$ under the same environmental conditions as described previously. Cold stress was performed by transferring the 12-d-old plants into the chamber at three different temperatures $\left(0,-2\right.$, and $\left.-4{ }^{\circ} \mathrm{C}\right)$ for $8 \mathrm{~h}$ and then returning to normal conditions for $5 \mathrm{~d}$.

\section{Results}

SEQUENCE ISOLATION AND ANALYSIS OF VVHOS1. A candidate gene fragment from $V$. vinifera with a length of 461 bp was selected from the Grape Genome Browser database. This fragment had high sequence identity with the AtHOS1 gene (At2g39810). Based on the RT-PCR and RACE analysis, a novel gene, designated VvHOS1 (KC524503), was isolated from 
'Muscat Hamburg'. The full length of VvHOS1 is 3118 bp with an open reading frame of 2931 bp and encodes a deduced protein of 976 amino acids with a theoretical isoelectric point of 5.74 and a molecular mass of $109.8 \mathrm{kDa}$.

VvHOS1 sequence homology was verified by using the BLAST algorithm on the NCBI server. The results revealed that VvHOS1 protein exhibits a high sequence identity with the HOS1-like proteins from other plant species such as arabidopsis (53\%, NP_181511), A. lyrata (56\%, XP_002879823), $P$. trichocarpa (64\%, XP_002304293), R. communis (63\%, EEF30910), P. trifoliata (64\%, ACY92092), O. sativa (56\%, AFK10207), and P. patens (41\%, XP_001780235).

Furthermore, a multiple sequence alignment was performed using the ClustalW program. Alignment analysis revealed that VvHOS1 protein contains a conserved RING finger domain in its N-terminal region (Supplementary Fig. 1). The RING finger motif can be described as a series of conserved cysteine and histidine residues and has a consensus sequence of $\mathrm{Cys}-\mathrm{X}_{2}$ Cys- $X_{(9-39)}-$ Cys- $X_{(1-3)}-$ His- $_{(2-3)}-$ Cys/His- $X_{2}-$ Cys- $X_{(4-48)^{-}}$ Cys- $\mathrm{X}_{2}-\mathrm{Cys}\left(\mathrm{C}_{3} \mathrm{HC}_{4}\right)$, where $\mathrm{X}$ can be any amino acid residue (Saurin et al., 1996). According to these results, we concluded that the predicted protein is the HOS1 protein from 'Muscat Hamburg'.

To investigate the phylogenetic relationship between VvHOS1 and other HOS1-like proteins, a phylogenetic tree was constructed (Supplementary Fig. 2) using the NJ method of the MEGA software (Version 4.0). The phylogenetic tree included three groups. The dicotyledonous species 'Muscat Hamburg', arabidopsis, A. lyrata, P. trichocarpa, R. communis, and $P$. trifoliata were clustered into the first group, and the monocotyledon $O$. sativa solely occupied the second group.
The non-vascular plant moss $P$. patens composed the third group. The phylogenetic tree suggested that VvHOS1 may have a closer relationship to the PtrHOS1 protein.

VVHOS1 EXPRESSION IS INDUCED BY COLD, DROUGHT, HIGH SALT, AND COLD PLUS DROUGHT CONDITIONS AS WELL AS EXOGENOUS ABA. To understand the function of $V v H O S 1$ better, we investigated $V v H O S 1$ transcript levels in shoot apices of 1-month-old 'Muscat Hamburg' seedlings, which had been subjected to exogenous $100 \mu \mathrm{M}$ ABA, or various stresses such as low temperature $\left(4^{\circ} \mathrm{C}\right)$, drought $(10 \%$ PEG 6000$)$, high salt $(100 \mathrm{~mm}$ $\mathrm{NaCl})$, and cold plus drought conditions $\left(4^{\circ} \mathrm{C}, 10 \%\right.$ PEG 6000). Real-time PCR analysis indicates that $V v H O S 1$ gene expression in 'Muscat Hamburg' could be induced by exogenous 100 ABA application and various abiotic stresses (Fig. 1). In response to cold stress, $V v H O S 1$ transcripts accumulation declined at $30 \mathrm{~min}$ after initiating cold stress; however, VvHOS1 expression had nearly recovered to its previous untreated level $(0 \mathrm{~h})$ at $1 \mathrm{~h}$ after cold stress. This rapid and transient reduction in VvHOS1 message in response to brief cold treatments was consistently observed in independent experiments. After recovering from the transient decrease, $V v H O S 1$ transcript level reached its maximal level, $2 \mathrm{~h}$ after the cold stress, expression decreased gradually to its minimum value by $24 \mathrm{~h}$, and recovered a little nearly to the untreated value at $48 \mathrm{~h}$ after treatment (Fig. 1A). After ABA treatment, $V v H O S 1 \mathrm{mRNA}$ transcripts increased slightly at $0.5 \mathrm{~h}$, decreased from 1 to $4 \mathrm{~h}$, and recovered nearly to the untreated level by 8,24 , and $48 \mathrm{~h}$ after ABA induction (Fig. 1B). VvHOS1 mRNA abundance declined at $0.5 \mathrm{~h}$ after exposure to drought conditions but later peaked with a 2 -fold increase over the untreated level after $1 \mathrm{~h}$; expression subsequently decreased gradually, reaching the minimum expression level at $4 \mathrm{~h}$; VvHOSI
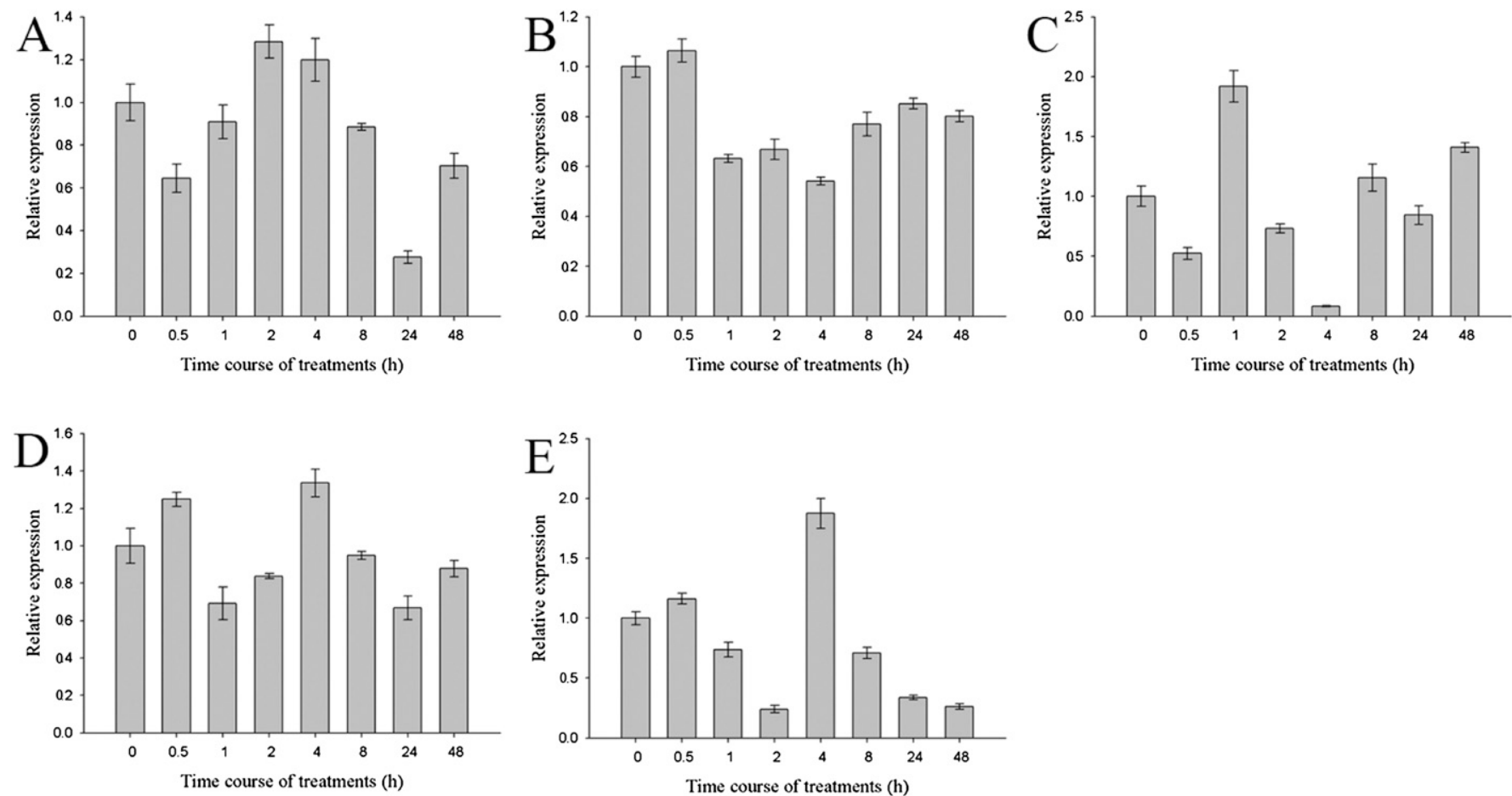

Fig. 1. Expression patterns of $V v H O S 1$ gene from 'Muscat Hamburg' grapevine in response to various treatments under a $24-\mathrm{h}$ light intensity of $100 \mu \mathrm{mol} \cdot \mathrm{m}^{-2} \cdot \mathrm{s}^{-1}$ : (A) low-temperature stress $\left(4^{\circ} \mathrm{C}\right),($ B) $100 \mu \mathrm{M}$ exogenous abscisic acid (ABA) for exogenous ABA induction, (C) drought stress [10\% polyethylene glycol (PEG) 6000], (D) salt stress (100 mM NaCl), and (E) $10 \%$ PEG 6000 at $4{ }^{\circ} \mathrm{C}$ mimicking cold plus drought condition. The expression level of $V v H O S 1$ gene in samples grown under normal growth conditions was set to 1 . The bars represent SD from three biological replicates. 


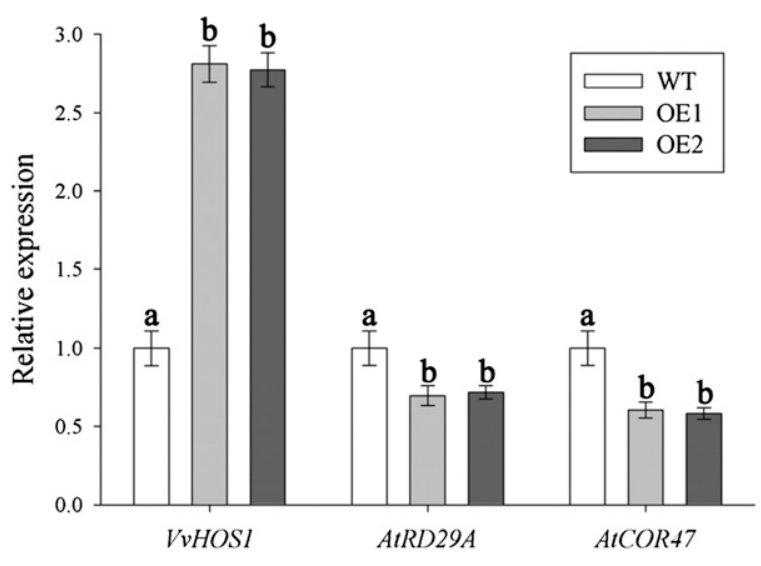

Fig. 2. Expression patterns of $V v H O S 1, A t R D 29 A$, and AtCOR47 in wild-type (WT) and transgenic arabidopsis plants (lines OE1 and OE2) under normal growth conditions. Parallel reactions using AtACTIN were carried out to normalize the amounts of added template. The expression level of each gene in the wild-type control was set as 1 . For $V v H O S 1$, the amplification on $10 \mathrm{pg}$ plasmid DNA (pCAMBIA 1301s-35S-VvHOSI) was set as 1 . The bars refer to SD of three biological replicates. Different upper case letters indicate significant differences at $P<0.05$ according to least significant difference multicomparison analysis.

mRNA expression levels returned to nearly the untreated level by $8 \mathrm{~h}$, declined slightly at $24 \mathrm{~h}$, and recovered to 1.5 -fold of the untreated level by the end of treatment (Fig. 1C). After salt treatment, $\mathrm{VvHOS} 1$ expression increased at $0.5 \mathrm{~h}$ and decreased at $1 \mathrm{~h}$; expression peaked at $4 \mathrm{~h}$ after treatment and gradually decreased by $24 \mathrm{~h}$; and expression recovered a little at the end (48 h; Fig. 1D). Cold plus drought stress induced the expression of $V v H O S 1$ slightly at $0.5 \mathrm{~h}$ after application of the stress; the expression then declined at $2 \mathrm{~h}$, peaked with a 2 -fold increase over the untreated level by $4 \mathrm{~h}$, and gradually decreased to minimum levels from 4 to $48 \mathrm{~h}$ (Fig. 1E).

Cold, drought, and SALT TOLERANCE OF TRANSGeniC ARABIDOPSIS PLANTS EXPRESSING VVHOS1. To assess the effect of $\mathrm{VvHOS} 1$ overexpression on endogenous gene expression, the expression of two stress response genes, AtRD29A and $\operatorname{AtCOR} 47$, were examined by real-time PCR in 4-week-old wild-type and $\mathrm{T}_{4}$ transgenic lines (OE1 and OE2) as shown in Figure 2. The expression of endogenous gene AtRD29A and AtCOR 47 was significantly lower in $V v H O S 1$-overexpression plants than wild-type arabidopsis under normal growth conditions. These results demonstrate that $\mathrm{VvHOS1}$ overexpression may have a negative impact on the expression of downstream stress response genes.

To investigate the biological function of $V v H O S 1$ gene in plant stress responses, two $\mathrm{T}_{4}$ transgenic lines (OE1 and $\mathrm{OE} 2$ ) overexpressing $\mathrm{V} v \mathrm{HOS} 1$ under the control of the $35 \mathrm{~S}$ promoter were selected to assess how $V v H O S 1$ affects cold, drought, or salt tolerance. Seeds from both wild-type and transgenic lines were grown on half-strength MS agar medium plates for $12 \mathrm{~d}$. A portion of the seedlings was exposed to three different temperatures $\left(0,-2\right.$, and $\left.-4^{\circ} \mathrm{C}\right)$ for $8 \mathrm{~h}$ and then grown in normal growth conditions for a further $5 \mathrm{~d}$ to assess their cold tolerance. The $V v H O S 1$-overexpression seedlings (OE1 and OE2) were less tolerant to low temperature than the wild type, particularly at $-4{ }^{\circ} \mathrm{C}$ (Fig. 3A-B). For example, exposure to $-4{ }^{\circ} \mathrm{C}$ killed nearly $55 \%$ of the transgenic lines (OE1 and OE2) but only $44 \%$ of wild-type plants. Another portion of the seedlings was transferred to half-strength MS medium plates supplemented with three different concentrations of PEG $6000(0 \%, 4 \%$, and $8 \%)$ or $\mathrm{NaCl}(0,20$, and $40 \mathrm{~mm})$. Five d after being exposed to drought or high salinity conditions, the growth of both the wild-type and transgenic lines were inhibited. However, the survival rate of wild-type plants was higher than that of transgenic plants [OE1 and OE2 (Fig. 3C-F)]. The lower survival rates of the VvHOS1expression lines (OE1 and OE2) were significantly different from that of the wild-type control (WT) after subjecting to low temperatures $\left(0,-2\right.$, and $\left.-4^{\circ} \mathrm{C}\right)$, different concentrations of PEG $6000(4 \%$ and $8 \%)$, and different concentrations of $\mathrm{NaCl}(20$ and $40 \mathrm{~mm})$. However, the survival rates of the $35 \mathrm{~S}-\mathrm{VvHOS} 1$ plants (OE1 and OE2) was not significantly different from that of the WT control under normal growth conditions (i.e., no PEG 6000 or $\mathrm{NaCl}$ ). These results show that the seedlings overexpressing VvHOS1 were less tolerant to low temperature, drought conditions, and high salt than the wild-type plants.

\section{Discussion}

'Muscat Hamburg' is a $V$. vinifera grape cultivar that was derived by crossing 'Schiava Grossa' with 'Muscat of Alexandria' (Crespan, 2003). 'Muscat Hamburg' is a black table grape that is highly appreciated for its beautiful bunches and its fair muscat flavor and can also be used to produce wine. For these valuable characteristics, breeders have used 'Muscat Hamburg' as a maternal parent to obtain new table grape cultivars. In this study, a negative regulator of cold-responsive genes, designated $V v H O S 1$, was identified from 'Muscat Hamburg' using RT-PCR and RACE methods. Multiple sequence alignments showed that VvHOS1 has a highly conserved RING finger domain in its N-terminal region (Supplementary Fig. 1). The RING finger motif is a small zinc-binding domain found in proteins of many eukaryotes. The proteins that contain this domain have been identified as functional E3 ubiquitin ligases, which interact with the E2 ubiquitin conjugating enzyme to degrade specific target proteins (Joazeiro and Weissman, 2000). In many metabolic processes, numerous proteins are ubiquitinated and degraded, which means that the existence of a large number of RING finger proteins may be needed to control the highly selective protein degradation process. For example, there are more than 1300 genes predicted to encode putative $\mathrm{E} 3$ subunits with one family containing almost 700 members (Smalle and Vierstra, 2004). As previously reported, the AtHOSI gene encodes a RING finger protein that functions as an E3 ubiquitin ligase; this ligase physically mediates the ubiquitination and degradation of ICE1 (Dong et al., 2006). Therefore, HOS1 acts as a negative regulator of cold-responsive genes because inactivation of ICE1 reduces the CBF-induced activation of many cold-responsive genes (Chinnusamy et al., 2003; Lee et al., 2005). VvHOS1 shared high sequence identity with the HOS1 protein from arabidopsis (53\%) according to the BLASTP analysis, suggesting that VvHOS1 may also interact with ICE1 and regulate the expression of cold-responsive genes. In addition, phylogenetic analysis shows that VvHOS1 is closely related to the HOS1-like proteins of $P$. trifoliata (Supplementary Fig. 2).

In this article, the expression of $V v H O S 1$ after different treatments was analyzed by real-time PCR. VvHOS1 expression was induced by low temperatures, as reported for HOS1 in arabidopsis, and the expression pattern observed for $\mathrm{VvHOSI}$ was similar to that of $\mathrm{AtHOS} 1$ when the plants were subjected to low temperatures (Lee et al., 2001). It is interesting that VvHOSI transcript abundance was transiently down-regulated 

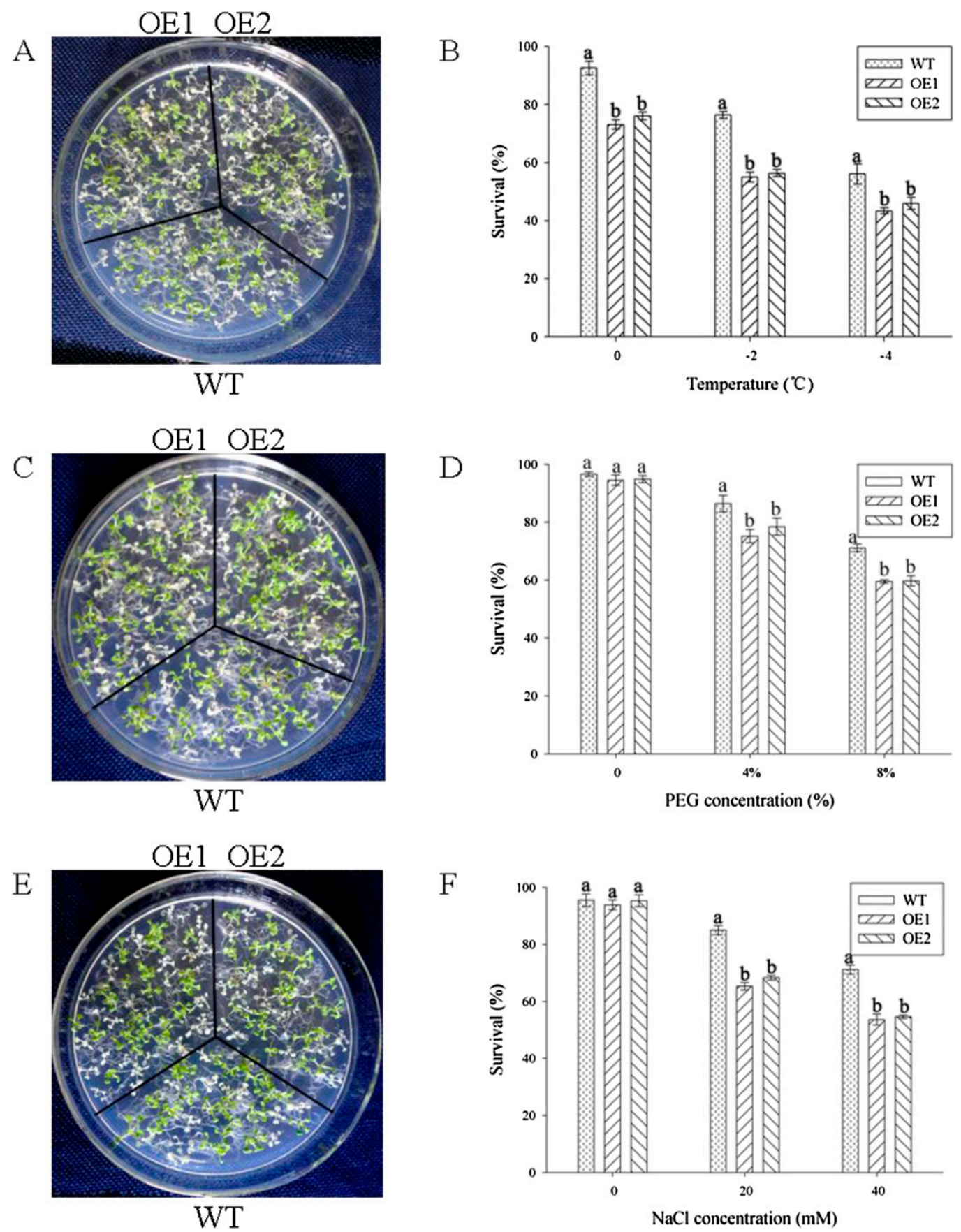

Fig. 3. Analysis of the stress tolerance and quantification of the survival rates for wild-type plants (WT) and transgenic arabidopsis plants overexpressing $V v H O S 1$. Twelve-d-old seedlings of the transgenic plants (OE1 and OE2) and the wild-type plants (WT) were subjected to $-4{ }^{\circ} \mathrm{C}$ for $8 \mathrm{~h}$ for cold stress and grown for a further $5 \mathrm{~d}$ in normal growth conditions (A), 8\% polyethylene glycol (PEG) 6000 for $5 \mathrm{~d}$ drought stress (C), and $40 \mathrm{~mm} \mathrm{NaCl}$ for $5 \mathrm{~d}$ salt stress (E). Quantification of the survival rates for WT and $V v H O S 1$-ovexpression transgenic plants ( $35 \mathrm{~S}-\mathrm{VvHOSI})$ after being subjected to 8 -h low-temperature treatment at $0,-2$, and $-4{ }^{\circ} \mathrm{C}$ (B); different concentrations of PEG $6000(0 \%, 4 \%$, and $8 \%)$ for drought stress (D); and different concentrations of $\mathrm{NaCl}(0,20$, and $40 \mathrm{~mm})$ for salt stress (F). The bars represent SD of three biological replicates, and different upper case letters indicate significant differences at $P<0.05$ according to least significant difference multicomparison analysis.

by cold stress (Fig. 1A). Because the genetic role of HOS1 is to attenuate cold signaling, the transient down-regulation of the HOS1 transcript may be important to allow the amplification of the cellular cold signals (Dong et al., 2006). During cold acclimation, the expression of a large number of genes is altered in plants (Benedict et al., 2006; Ishitani et al., 1998; Lee et al., 2001; Thomashow, 1999). This rapid transient reduction in expression and the extended duration of $V v H O S 1$ expression in response to cold treatment suggests that $V v H O S 1$ may help activate the cold-responsive genes during cold acclimation in grape, as previously reported (Dong et al., 2006; Lee et al., 2001).

The transcript levels of $V v H O S 1$ also fluctuated after ABA application or after exposure to drought or high salinity (Fig. 1B-D); this expression pattern differs from that of AtHOS1. Although AtHOS1 transcript abundance was not substantially 
influenced by ABA, $\mathrm{NaCl}$, or PEG treatments, the expression patterns of AtHOS1 under these stresses were also unknown (Lee et al., 2001). To date, no study has focused on the effects of cold plus drought conditions on the gene expression patterns in grape. Thus, we analyzed the expression of $V v H O S 1$ under cold plus drought conditions; this stress is the most common one that grape encounters during winter in the major grape-producing areas in northern China. The results of the real-time PCR analysis suggest that cold plus drought conditions could also induce $V v H O S 1$ expression (Fig. 1E). Based on these findings, we suggest that $V v H O S 1$ expression is also regulated by exogenous ABA as well as drought, salt, and cold plus drought conditions, indicating that there is substantial cross-talk in the various stress signal transduction pathways in plants. In short, VvHOS1 may be involved in ABA-dependent and ABAindependent signal transduction pathways and thereby affects the stress response of 'Muscat Hamburg'.

Ectopic expression of $V v H O S 1$ resulted in decreased survival of the transgenic plants $(35 S-V v H O S 1)$ after exposure to freezing temperatures, which was similar to the effects of overexpressing AtHOS1 in arabidopsis (Dong et al., 2006). Meanwhile, the expression levels of AtRD29A and AtCOR47 were also decreased in $35 S-V v H O S 1$ plants. Because the arabidopsis RING E3 ligase RGLG2, DRIP1, and DRIP2 proteins all negatively regulate the plant drought stress response (Cheng et al., 2012; Qin et al., 2008), it is possible that the overexpression of $V v H O S 1$ may reduce a plant's tolerance to drought. The sensitivity of the 35S: $V v H O S 1$ plants to salt stress is similar to that of transgenic arabidopsis overexpressing the RING-H2 gene AtXERICO during early seedling growth (Ko et al., 2006). In this experiment, all of the $\mathrm{T}_{4}$ transgenic plants tested (Fig. 3) were hypersensitive to cold, drought, and salt stresses compared with the WT plants.

Our findings indicate that expression of VvHOS1 can be induced by a variety of abiotic stress conditions and the application of the signaling molecule ABA. Ectopic expression of $V v H O S 1$ in arabidopsis plants decreased tolerance to cold, drought, and salt stress and suppressed the expression of AtRD29A and $A t C O R 47$. In conclusion, $V v H O S 1$ may be a negative regulator of the stress response in 'Muscat Hamburg'. Further investigations are necessary to understand the exact nature of the function of VvHOS1 protein and the effects of its overexpression on more cold-responsive genes, which may provide a novel approach to regulating plants' tolerance to different environmental stresses.

\section{Literature Cited}

Benedict, C., J.S. Skinner, R. Meng, Y. Chang, R. Bhalerao, N.P.A. Huner, C.E. Finn, T.H.H. Chen, and V. Hurry. 2006. The CBF1dependent low-temperature signalling pathway, regulon and increase in freeze tolerance are conserved in Populus spp. Plant Cell Environ. 29:1259-1272.

Cheng, M.C., E.J. Hsieh, J.H. Chen, H.Y. Chen, and T.P. Lin. 2012. Arabidopsis RGLG2, functioning as a RING E3 ligase, interacts with AtERF53 and negatively regulates the plant drought stress response. Plant Physiol. 158:363-375.

Chinnusamy, V., M. Ohta, S. Kanrar, B.H. Lee, X. Hong, M. Agarwal, and J.K. Zhu. 2003. ICE1: A regulator of cold-induced transcriptome and freezing tolerance in Arabidopsis. Genes Dev. 17:10431054.

Clough, S.J. and A.F. Bent. 1998. Floral dip: A simplified method for Agrobacterium-mediated transformation of Arabidopsis thaliana. Plant J. 16:735-743.
Crespan, M. 2003. The parentage of Muscat of Hamburg. Vitis 42: 193-197.

Dong, C.H., M. Agarwal, Y. Zhang, Q. Xie, and J.K. Zhu. 2006. The negative regulator of plant cold responses, HOS1, is a RING E3 ligase that mediates the ubiquitination and degradation of ICE1. Proc. Natl. Acad. Sci. USA 103:8281-8286.

Fennell, A. 2004. Freezing tolerance and injury in grapevines. J. Crop Improv. 10:201-235.

Fursova, O.V., G.V. Pogorelko, and V.A. Tarasov. 2009. Identification of ICE2, a gene involved in cold acclimation which determines freezing tolerance in Arabidopsis thaliana. Gene 429:98-103.

Gamborg, O.L., R.A. Miller, and K. Ojima. 1968. Nutrient requirements of suspension cultures of soybean root cells. Exp. Cell Res. 50:151-158.

Ganeshan, S., P. Vitamvas, D.B. Fowler, and R.N. Chibbar. 2008. Quantitative expression analysis of selected COR genes reveals their differential expression in leaf and crown tissues of wheat (Triticum aestivum L.) during an extended low temperature acclimation regimen. J. Expt. Bot. 59:2393-2402.

Genoscope. 2009. Grape genome browser (12X), Blast-Search. 1 June 2010. $<\mathrm{http}: / /$ www.genoscope.cns.fr/cgi-bin/blast_server/projet_ ML/blast.pl>.

Guy, C.L. 1990. Cold acclimation and freezing stress tolerance: Role of protein metabolism. Annu. Rev. Plant Biol. 41:187-223.

Hellemans, J., G. Mortier, A. De Paepe, F. Speleman, and J. Vandesompele. 2007. qBase relative quantification framework and software for management and automated analysis of real-time quantitative PCR data. Genome Biol. 8:R19.

Ishitani, M., L. Xiong, H. Lee, B. Stevenson, and J.K. Zhu. 1998. HOS1, a genetic locus involved in cold-responsive gene expression in Arabidopsis. Plant Cell 10:1151-1161.

Joazeiro, C.A. and A.M. Weissman. 2000. RING finger proteins: Mediators of ubiquitin ligase activity. Cell 102:549-552.

Knight, H., S.G. Mugford, B. Ulker, D. Gao, G. Thorlby, and M.R. Knight. 2009. Identification of SFR6, a key component in cold acclimation acting post-translationally on CBF function. Plant J. 58:97-108.

Ko, J.H., S.H. Yang, and K.H. Han. 2006. Upregulation of an Arabidopsis RING-H2 gene, XERICO, confers drought tolerance through increased abscisic acid biosynthesis. Plant J. 47:343-355.

Lazaro, A., F. Valverde, M. Pineiro, and J.A. Jarillo. 2012. The Arabidopsis E3 ubiquitin ligase HOS1 negatively regulates CONSTANS abundance in the photoperiodic control of flowering. Plant Cell 24:982-999.

Lee, B.H., D.A. Henderson, and J.K. Zhu. 2005. The Arabidopsis coldresponsive transcriptome and its regulation by ICE1. Plant Cell 17: 3155-3175.

Lee, C.M. and M.F. Thomashow. 2012. Photoperiodic regulation of the $\mathrm{C}$-repeat binding factor $(\mathrm{CBF})$ cold acclimation pathway and freezing tolerance in Arabidopsis thaliana. Proc. Natl. Acad. Sci. USA 109:15054-15059.

Lee, H., L. Xiong, Z. Gong, M. Ishitani, B. Stevenson, and J.K. Zhu. 2001. The Arabidopsis HOS1 gene negatively regulates cold signal transduction and encodes a RING finger protein that displays coldregulated nucleo-cytoplasmic partitioning. Genes Dev. 15:912-924. Levitt, J. 1980. Responses of plants to environmental stresses. Academic Press, London, UK.

Medina, J., R. Catala, and J. Salinas. 2011. The CBFs: Three Arabidopsis transcription factors to cold acclimate. Plant Sci. 180:3-11.

Michaels, S.D. and R.M. Amasino. 1999. Flowering locus C encodes a novel MADS domain protein that acts as a repressor of flowering. Plant Cell 11:949-956.

Miura, K., J.B. Jin, J. Lee, C.Y. Yoo, V. Stirm, T. Miura, E.N. Ashworth, R.A. Bressan, D.J. Yun, and P.M. Hasegawa. 2007. SIZ1mediated sumoylation of ICE1 controls CBF3/DREB1A expression and freezing tolerance in Arabidopsis. Plant Cell 19:1403-1414.

Mullins, M.G., A. Bouquet, and L.E. Williams. 1992. Biology of the grapevine. Cambridge Univ. Press, London, UK. 
Murashige, T. and F. Skoog. 1962. A revised medium for rapid growth and bio assays with tobacco tissue cultures. Physiol. Plant. 15:473497.

Orvar, B.L., V. Sangwan, F. Omann, and R.S. Dhindsa. 2000. Early steps in cold sensing by plant cells: The role of actin cytoskeleton and membrane fluidity. Plant J. 23:785-794.

Provart, N.J., P. Gil, W. Chen, B. Han, H.S. Chang, X. Wang, and T. Zhu. 2003. Gene expression phenotypes of Arabidopsis associated with sensitivity to low temperatures. Plant Physiol. 132:893-906.

Qin, F., Y. Sakuma, L.S. Tran, K. Maruyama, S. Kidokoro, Y. Fujita, M. Fujita, T. Umezawa, Y. Sawano, K. Miyazono, M. Tanokura, K. Shinozaki, and K. Yamaguchi-Shinozaki. 2008. Arabidopsis DREB2A-interacting proteins function as RING E3 ligases and negatively regulate plant drought stress-responsive gene expression. Plant Cell 20:1693-1707.

Ruijter, J.M., C. Ramakers, W.M.H. Hoogaars, Y. Karlen, O. Bakker, M.J.B. van den Hoff, and A.F.M. Moorman. 2009. Amplification efficiency: Linking baseline and bias in the analysis of quantitative PCR data. Nucleic Acids Res. 37:e45.

Saurin, A.J., K. Borden, M.N. Boddy, and P.S. Freemont. 1996. Does this have a familiar RING? Trends Biochem. Sci. 21:208.

Schultz, J., F. Milpetz, P. Bork, and C.P. Ponting. 1998. SMART, a simple modular architecture research tool: Identification of signaling domains. Proc. Natl. Acad. Sci. USA 95:5857-5864.

Sheldon, C.C., D.T. Rouse, E.J. Finnegan, W.J. Peacock, and E.S. Dennis. 2000. The molecular basis of vernalization: The central role of Flowering Locus C (FLC). Proc. Natl. Acad. Sci. USA 97:37533758 .

Smalle, J. and R.D. Vierstra. 2004. The ubiquitin 26S proteasome proteolytic pathway. Annu. Rev. Plant Biol. 55:555-590.

Suzuki, I., D.A. Los, and N. Murata. 2000. Perception and transduction of low-temperature signals to induce desaturation of fatty acids. Biochem. Soc. Trans. 28:628-630.
Suzuki, N. and R. Mittler. 2005. Reactive oxygen species and temperature stresses: A delicate balance between signaling and destruction. Physiol. Plant. 126:45-51.

Swiss Institute of Bioinformatics. 1993. ExPASy bioinformatics resource portal, translate. 1 July $2010 .<$ http://web.expasy.org/translate/>.

Tamura, K., J. Dudley, M. Nei, and S. Kumar. 2007. MEGA4: Molecular evolutionary genetics analysis (MEGA) software version 4.0. Mol. Biol. Evol. 24:1596-1599.

Tang, K.X., X.L. Wang, X.F. Sun, and Z.X. Deng. 2006. Cold: A double-edged sword to plants, p. 102-107. In: Teixeira da Silva, J.A. (ed.). Floriculture, ornamental and plant biotechnology. Global Science Books, Isleworth, UK.

Thiel, G., M. Lietz, and M. Hohl. 2004. How mammalian transcriptional repressors work. Eur. J. Biochem. 271:2855-2862.

Thomashow, M.F. 1999. Plant cold acclimation: Freezing tolerance genes and regulatory mechanisms. Annu. Rev. Plant Physiol. Plant Mol. Biol. 50:571-599.

Thomashow, M.F. 2010. Molecular basis of plant cold acclimation: Insights gained from studying the CBF cold response pathway. Plant Physiol. 154:571-577.

Thompson, J.D., D.G. Higgins, and T.J. Gibson. 1994. CLUSTAL $\mathrm{W}$ : Improving the sensitivity of progressive multiple sequence alignment through sequence weighting, position-specific gap penalties and weight matrix choice. Nucleic Acids Res. 22:4673-4680.

Xiao, H., M. Siddiqua, S. Braybrook, and A. Nassuth. 2006. Three grape CBF/DREB1 genes respond to low temperature, drought and abscisic acid. Plant Cell Environ. 29:1410-1421.

Yan, J., J. Wang, Q. Li, J.R. Hwang, C. Patterson, and H. Zhang. 2003. AtCHIP, a U-box-containing E3 ubiquitin ligase, plays a critical role in temperature stress tolerance in Arabidopsis. Plant Physiol. 132:861-869.

Zarka, D.G., J.T. Vogel, D. Cook, and M.F. Thomashow. 2003. Cold induction of Arabidopsis CBF genes involves multiple ICE (inducer of $\mathrm{CBF}$ expression) promoter elements and a cold-regulatory circuit that is desensitized by low temperature. Plant Physiol. 133:910-918. 

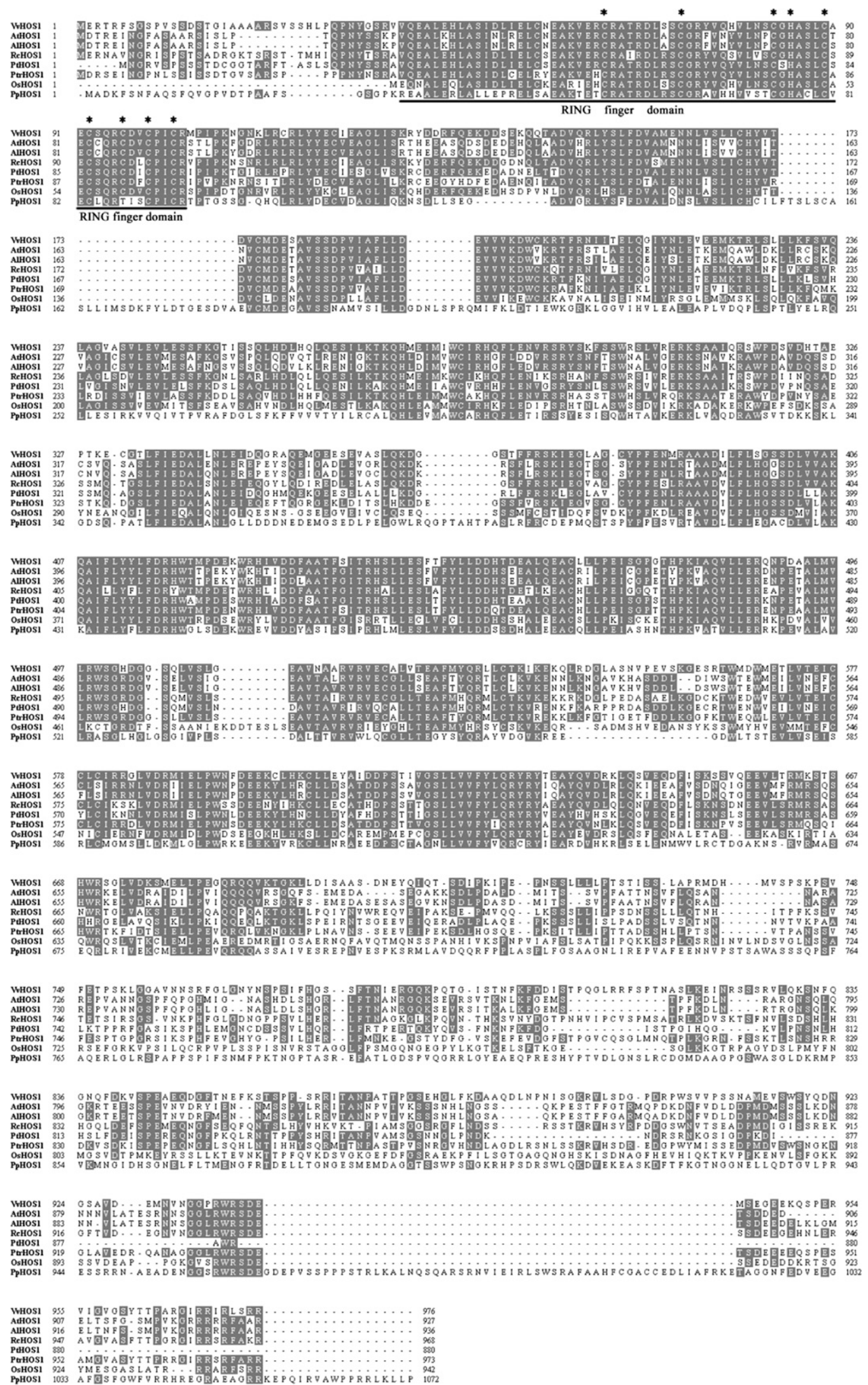

Supplementary Fig. 1. Amino acid sequence alignment of the HOS1-like proteins from Arabidopsis thaliana, Arabidopsis lyrata, Populus trichocarpa, Ricinus communis, Poncirus trifoliata, Oryza sativa, Physcomitrella patens, and Vitis vinifera 'Muscat Hamburg'. Identical amino acids are shaded. The conserved RING finger domain is underlined. The conserved cysteine and histidine residues are indicated by asterisks. 


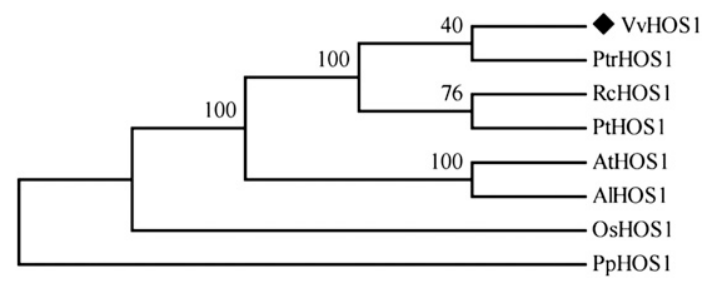

Supplementary Fig. 2. The phylogenetic tree of the close homologues of PtrHOS1 and other HOS1-like proteins found in plants. The solid diamond represents VvHOS1. The polypeptides used in the analysis include VvHOS1 from Vitis vinifera 'Muscat Hamburg' (AGH20655.1), AtHOS1 from Arabidopsis thaliana (NP_181511), AlHOS1 from Arabidopsis lyrata (XP_002879823), PtHOS1 from Populus trichocarpa (XP_002304293), RcHOS1 from Ricinus communis (EEF30910), PtrHOS1 from Poncirus trifoliata (ACY92092), OsHOS1 from Oryza sativa (AFK10207), and PpHOS1 from Physcomitrella patens (XP_001780235). Construction of the phylogenetic tree was performed using the neighbor-joining algorithm in MEGA 4.0 software. The numbers at each node represent the bootstrap values for 1000 replicates. 